\title{
The lifetime of contact binaries
}

\author{
D. K. Jiang ${ }^{1,2}$ Z. W. Han ${ }^{1,2}$, L. H. Yang ${ }^{3}$ and L. F. Li ${ }^{1,2}$ \\ ${ }^{1}$ National Astronomical Observatories, Yunnan Observatory, Chinese Academy of Sciences, \\ P.O. Box 110, Kunming. e-mail: dengkai@ynao.ac.cn \\ ${ }^{2}$ Key Laboratory for the Structure and Evolution of Celestial Objects, Chinese Academy of \\ Sciences \\ ${ }^{3}$ National Astronomical Observatories, Chinese Academy of Sciences, Beijing 100012, China
}

\begin{abstract}
The lifetime of contact binaries is an important parameter for studying the evolution of contact binaries. By reproducing the relative frequency of occurrence for contact binaries, we found that the lifetime of contact binaries decreases with increasing mass of the primaries. The lifetime of contact binaries is about $4 \%-10 \%$ of the main sequence lifetime of the primaries, and is about 0.006-0.65 Gyr. This suggests that the lifetime of contact binaries strongly depends on the mass of the primaries.
\end{abstract}

Keywords. Binaries: close, binaries: eclipsing, stars: evolution

\section{Introduction}

Contact binaries are interacting binaries that two components are overflowing their own Roche lobe. The lifetime of contact binaries $\left(\tau_{\mathrm{c}}\right.$, a timescale from the formation of contact binary to its merger) is an important parameter for studying the evolution of contact binaries and their products (e.g. blue stragglers, FK Comae type stars, and $\lambda$ Bootis type stars). However, the lifetime of contact binaries is still an open issue. According to the relative frequency of occurrence (RFO) for contact binaries, Vilhu (1982) found that the lifetime of contact binaries with $M_{1}=1.5 \mathrm{M}_{\odot}$ is about 0.5 Gyr. Bilir et al. (2005) suggested that a difference of kinematical age $(1.61 \mathrm{Gyr})$ between the field contact binaries and the field chromospherically active binaries is regarded as a mean lifetime of contact binaries. However, it is uncertain that how the lifetime of contact binaries depends on the different primaries. In this study, we attempt to find the lifetime of contact binaries with different primaries by reproducing the RFO for contact binaries.

\section{Computations and results}

The RFO $\left(r_{\mathrm{RFO}}\right)$ is a number ratio of contact binaries to single main sequence (MS) stars with the same spectral type, which is about $0.2 \%$ (Rucinski 2006). For a contact binaries with a given primary mass, the RFO can be expressed as $r_{\mathrm{RFO}} \simeq\left(N_{\mathrm{c}} \times \tau_{\mathrm{c}}\right) /\left(N_{\mathrm{s}} \times\right.$ $\tau_{\mathrm{ms}}$ ), where $N_{\mathrm{c}}, \tau_{\mathrm{c}}$ is the number and lifetime of contact binaries, and $N_{\mathrm{s}}, \tau_{\mathrm{ms}}$ are the number and lifetime of single MS stars that have same spectral type as contact binaries. Then, the lifetime of contact binaries is $\tau_{\mathrm{c}} \simeq \alpha_{\mathrm{c}} \times \tau_{\mathrm{ms}}=r_{\mathrm{RFO}} \times\left(N_{\mathrm{s}} / N_{\mathrm{c}}\right) \times \tau_{\mathrm{ms}}$. The ratio $\alpha_{\mathrm{c}}$ represents the ratio of the lifetime of contact binaries to the MS lifetime of the primaries of contact binaries. We assume that these single stars with the same mass as the primaries of contact binaries have the same spectral type as contact binaries. To obtain $N_{\text {c }}$ and $N_{\mathrm{s}}$, we performed a series of detailed Monte Carlo simulations with the Hurley's rapid binary evolution code (Hurley, Tout \& Pols 2002). We follow the evolution of $10^{6}$ sample binaries (metallicity $\mathrm{Z}=0.02$ ) and adopt the following input for the simulations (see Han 1998, for details). (1) The initial mass function (IMF) of Miller \& Scalo (1979) 

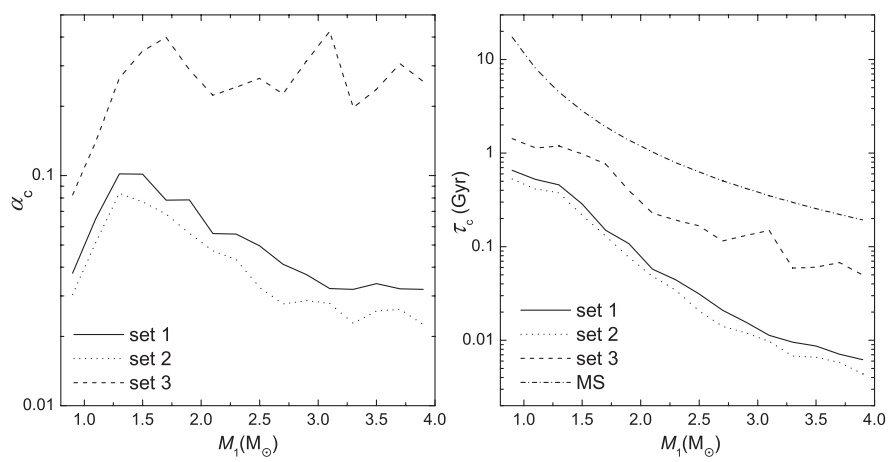

Figure 1. The ratio $\alpha_{\mathrm{c}}$ (left-hand panel) and lifetime $\tau_{\mathrm{c}}$ of contact binaries (right-hand panel). Set 1 , set 2 and set 3 are a constant mass ratio distribution, a rising mass ratio distribution and a independent mass ratio distribution. The dash-dot line represents the lifetime of MS for the primaries.

is adopted. (2) The mass-ratio distribution is taken to be constant (Set 1), a rising mass ratio distribution (Set 2) or, alternatively, it is assumed that the component masses are uncorrelated (Set 3). (3) We take the distribution of separations to be constant in log a for wide binaries, where a is the orbital separation. (4) The orbits are assumed to be circular.

The ratio $\alpha_{\mathrm{c}}$ depends on the mass of the primaries and has a range from 0.04 to 0.1 (left-hand panel of Figure 1, set 1). This means that the lifetime of contact binaries is about $4 \%-10 \%$ of the MS lifetime of the primaries. The ratio for the rising mass ratio distribution (set 2) is close to that for the constant mass ratio distribution. However, the ratio for the independent mass ratio distribution (set 3) is larger than that for other distributions, because there are more systems with very small mass ratio that could not evolve into contact. The lifetime of contact binaries decreases from 0.65 Gyr for $M_{1} \sim 0.9 \mathrm{M}_{\odot}$ to $0.006 \mathrm{Gyr}$ for $M_{1} \sim 3.9 \mathrm{M}_{\odot}$ (right-hand panel of Figure 1, set 1 ). Therefore, the lifetime of contact binaries also depends on the primary mass.

\section{Conclusions}

We investigated the lifetime of contact binaries by reproducing the RFO for contact binaries and found that the lifetime of contact binaries decreases with increasing mass of the primaries. This suggests that the lifetime of contact binaries strongly depends on the mass of the primaries.

\section{References}

Bilir, S., Karatas, Y., Demircan, O., \& Eker, Z. 2005, MNRAS, 357, 497

Han, Z. 1998, MNRAS, 296, 1019

Hurley, J. R., Tout, C. A., \& Pols, O. R. 2002, MNRAS, 329, 897

Miller, G. E. \& Scalo, J. M. 1979, ApJSs, 41, 513

Rucinski, S. M. 2006, MNRAS, 368, 1319

Vilhu, O. 1982, $A \mathscr{E} A$ A, 109, 17 